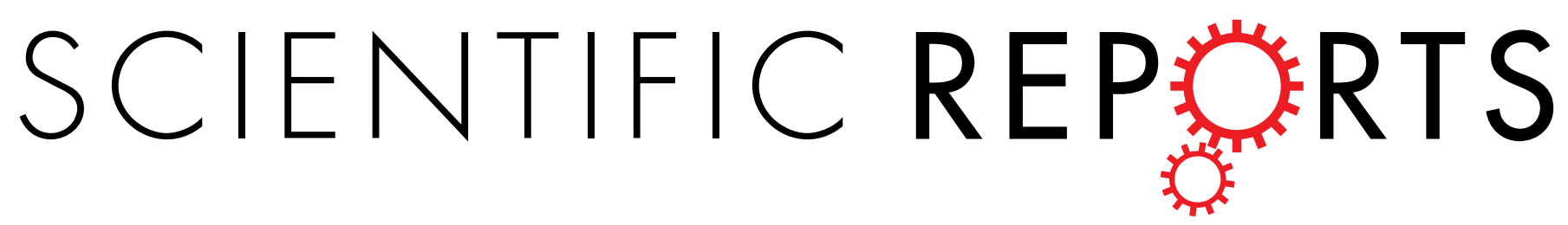

\title{
Erratum: The up-scaling of ecosystem functions in a heterogeneous world
}

Andrew M. Lohrer, Simon F. Thrush, Judi E. Hewitt \& Casper Kraan

Scientific Reports 5:10349; doi: 10.1038/srep10349; published online 20 May 2015; updated on 31 July 2015

In the original version of this Article, the Abstract contains a typographical error.

"Additional experimentation illustrated how these effects amplified over time, resulting in greater primary producer biomass sediment chlorophyll $a$ content (Chla) in the longer term".

should read:

"Additional experimentation illustrated how these effects amplified over time, resulting in greater primary producer biomass (sediment chlorophyll $a$ content) in the longer term".

This has now been corrected in both the PDF and HTML versions of the Article. 